\title{
Formation, migration, and stability of extrasolar planetary systems
}

\author{
Fred C. Adams \\ Michigan Center for Theoretical Physics, Department of Physics \\ University of Michigan, Ann Arbor, MI 48109 USA \\ email: fca@umich.edu
}

\begin{abstract}
This paper presents recent results concerning the planet formation, planet migration, and the long term stability of planetary systems. Most stars are found in binary systems and binary companions can disrupt both planet formation and stability. We first consider the effects of outer binary companions on the late stages of terrestrial planet formation and show how planet formation depends on the binary periastron. We then consider migration mechanisms for giant planets. In this case, planet scattering produces the full range of orbital eccentricities, but is less effective in moving planets inward (decreasing their semi-major axes). Disk torques are effective at moving planets inward, but not at increasing the eccentricities. We explore a scenario in which disk torques act in concert with planet scattering to provide the full range of orbital elements observed in extrasolar planetary systems. Finally, we consider the longer term stability of Earth-like planets in binary systems; we find that nearly 50 percent of binaries allow for Earth-like planets to remain stable over the current (4.6 Gyr) age of our solar system.
\end{abstract}

Keywords. Extrasolar planets, planetary dynamics, planet formation

\section{Introduction}

During the course of planet formation, planet migration, and the subsequent evolution of planetary systems, chaos plays an important role in the underlying dynamics. In particular, all of these systems exhibit sensitive dependence on their initial conditions, so that the result of any given process cannot be unambiguously predicted. Instead, the results must be described in terms of the distributions of possible outcomes. In this contribution, we discuss a collection of subproblems in planet formation/migration where the systems display sensitive dependence on the initial conditions. During terrestrial planet formation, the locations, masses, and numbers of planets in the resulting solar system must be described as a distribution. In this context, we consider the late stages of terrestrial planet formation in binary star systems and show that the planet formation process leads to a wide distribution of outcomes, but well-defined trends can still be determined (Figure 1). For example, we can determine the fraction of binary systems that allow the terrestrial planet formation to take place unimpeded. When planets migrate, the orbital elements of the final state display a distribution of values. Here we explore a scenario for giant planet migration in which disk torques act to move the planets inward and planetplanet scattering acts to increase the orbital eccentricities; the resulting distributions of orbital elements predicted by the theory are in reasonable agreement with those of observed extrasolar planets (Figure 2). Finally, planets on unstable orbits in binary star systems display a wide distribution of ejection times (Figure 3). Nonetheless, the distributions of ejection times exhibit well-defined dependences on the binary parameters and we can determine the fraction of binary systems that allow Earth-like planets to remain stable over the current age of the solar system. 


\section{Terrestrial Planet Formation in Binary Systems}

The first part of this work studies the formation of terrestrial planets in binary star systems, with the goal of quantifying the effects of the binary companions on the planet formation process. We note that the extrasolar planets discovered thus far (e.g., Mayor \& Queloz 1995; Marcy \& Butler 1996; Butler et al. 1999; Marcy et al. 2001) have masses near that of Jupiter and are thus thought to be gaseous giant planets. Although terrestrial planets have not been detected in extrasolar systems with main-sequence primaries due to their small masses, they are expected to readily form in such systems alongside their Jovian counterparts (e.g., Lissauer 1993). Indeed, the following three lines of evidence suggest that terrestrial planets might be common: (1) Our solar system has produced terrestrial planets, and no instances of astronomical creation are known to be unique. (2) Our solar system has manufactured a large number of moons, asteroids, and other rocky bodies that presumably formed in the same manner as the planets. (3) Terrestrial planets have been discovered in orbit around the pulsar PSR $1257+12$ and hence were able to form in a harsh environment (Wolszczan \& Frail 1992).

In order to study the formation of terrestrial planets, we have performed a series of $N$-body simulations using the Mercury integration package (Chambers 1999), which has recently been modified to include a stellar binary companion (Chambers et al. 2002). These computations follow the evolution of a field of planetessimals as they evolve into a terrestrial planet system (Quintana 2004; Quintana et al. 2004). The simulations start with 14 planetary embryos (with mass $5.6 \times 10^{26} \mathrm{~g}$ ) and 140 planetessimals with a mass ten times smaller (each population of starting bodies thus accounts for half of the mass). The bodies are initially distributed on nearly circular orbits with semi-major axes in the range $0.36 \mathrm{AU} \leqslant a \leqslant 2.05 \mathrm{AU}$. This particular set of initial conditions was chosen because it tends to produce terrestrial planet systems like our own when implemented around a single star of one solar mass (Chambers 2001). The simulations run for approximately $100 \mathrm{Myr}$ and evolve into terrestrial planet systems with a wide range of orbital elements and a wide range of planet masses.

These simulations are idealized in that they consider only the late stages of planetessimal accumulation, when planets are assembled from hundreds of smaller rocky bodies. As a result, these simulations do not provide a definitive determination of the full range of terrestrial planet configurations that are possible. However, the part of the problem that we understand best is the orbital dynamics and our codes properly integrate the known equations of motion. In particular, the perturbations due to the binary companion are well-modeled, so we can achieve an accurate representation of the binary effects within this idealized scenario for terrestrial planet formation.

Thus far, we have run $\sim 100$ simulations of terrestrial planet formation using equal mass binaries with varying orbital elements, in particular the semi-major axis $a_{b}$ and orbital eccentricity $\epsilon_{b}$ (Quintana 2004; Quintana et al. 2004; Lissauer et al. 2004). One set of results is shown in Figure 1. Like most dynamical systems of this type, these systems experience chaotic behavior and exhibit sensitive dependence on the initial conditions. In the present context, for example, moving one planetessimal forward in its orbit by one meter (at the start of the simulation) can lead to a change in the number of planets produced at the end of the simulation. As a result, most quantities of interest (e.g., the number of planets produced or their orbital elements) cannot be described by a single value, but rather by a full distribution, even for effectively equivalent starting conditions. Although a study of the full parameter space for planet formation in binary systems is just beginning, the importance of presenting the results in terms of distributions is already apparent (see also Levison et al. 1998). As shown in Figure 1, for the set of simulations 


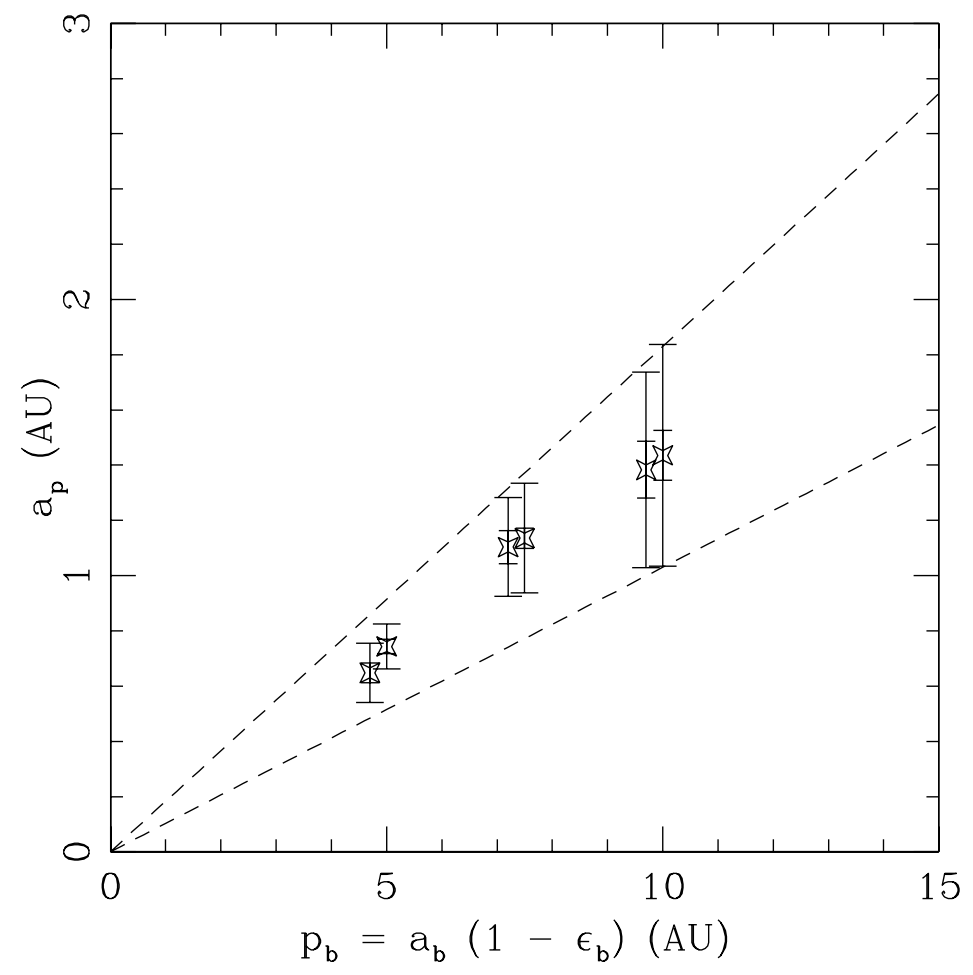

Figure 1. The effect of binary companions on the location of the outermost planet in numerical simulations of terrestrial planet formation in binary systems (Quintana et al. 2004). The semi-major axis $a_{p}$ of the outermost terrestrial planet is plotted as a function of the periastron distance $p_{b}$ for the binary. The mass ratio of the binary is taken to be unity for all cases shown; the stellar masses are $1.0 M_{\odot}$ and $0.5 M_{\odot}$ for the pairs of data points shown here. For this ensemble of simulations, the location of the outermost terrestrial planet takes on a distribution of values, with the width of the distribution indicated by the larger error bars. The smaller (inside) error bars indicate the effective error in determining the mean value. The dashed lines bracket the range of planet location as a function of binary periastron.

with a given value of periastron $p_{b}$, we find a wide range of values for the location $a_{p}$ of the outermost terrestrial planet, where the range is depicted by the large error bars in the figure. Nonetheless, well defined trends emerge. In this case, the distribution of terrestrial system sizes $\left(a_{p}\right)$ is a function of periastron: The range of planet locations (the values of $a_{p}$ for the outermost planet) gets smaller and the mean value systematically decreases as the binary periastron $p_{b}$ grows smaller. Further, although the initial field of rocky building blocks extends out to 2.05 AU, only a few planets are formed with semi-major axis near $2 \mathrm{AU}$ and none of the simulations produce planets beyond 2 AU. The results shown here (from Quintana et al. 2004) thus demonstrate how binary companions can limit the growth of terrestrial planets (see also Levison \& Agnor 2003) and demonstrate that many realizations of the problem must be considered in order to sample the full distribution of outcomes. 
The results shown in Figure 1 indicate that when binary periastron $p_{b}<10 \mathrm{AU}$, the binary companion limits the radial extent of the terrrestrial planet region. For sufficiently wide binaries (large peristron values), however, the binary will have minimal effect on the formation of terrestrial planets. In Figure 1, the lower dashed curve, which tracks the lower end of the $a_{p}$ range, can be extrapolated out to where it exceeds the extent of our terrestrial planet region $\left(a_{p} \approx 2 \mathrm{AU}\right)$, which occurs at $p_{b} \approx 19$ AU. Using this extrapolation, our results obtained to date suggest that binaries with periastron $p_{b}>19$ AU have little effect on terrestrial planet formation (although more simulations and hence better statistics would be desirable). Given that more than two thirds of stars are found in binary systems (Abt 1983), what fraction of binaries are sufficiently wide, with $p_{b}>19$ AU, so that terrestrial planet formation is only minimally affected?

To answer this question, we need to find the fraction of binaries with periastron greater than a set value $p$, as a function of $p$. The orbital elements of binaries have reasonably well measured distributions (Duquennoy \& Mayor 1991; hereafter DM91). The binary population has a period distribution with a log-normal form; for primary stars with $M_{*} \sim$ $1 M_{\odot}$, we can convert the period distribution into the distribution of semi-major axes $a_{b}$ (using the measured distribution of the mass ratio $\mu=M_{C} / M_{*}$ - again see DM91). The eccentricity distribution is also observed, and is independent of semi-major axis for the wide binaries of interest here (DM91). By integrating over the portion of binary parameter space corresponding to periastron greater than a set value $p$, we numerically obtain the fraction $F(p)$ of binaries with $p_{b} \geqslant p$ (David et al. 2003). A fitting function to the numerically determined result can be written in the simple form

$$
F(p)=F_{1} \exp \left[-\left(a \xi+b \xi^{2}\right)\right]
$$

where $F_{1}=0.711, a=0.101, b=0.0287$, and $\xi \equiv \ln [p /(1 \mathrm{AU})]$. The function [2.1] provides a good approximation to the numerically determined result, with an absolute error less than about 0.011 and a relative error less than 4 percent. More exact fits are not warranted, as the observed distributions of binary orbital parameters are not known to this accuracy. This function can be used to estimate the fraction of binary systems with periastron greater than any specified value within the allowed range $1<p<10^{5}$. In the present context we find that about 40 percent of binaries have periastron $p_{b} \geqslant 19$ $\mathrm{AU}$ and hence have little effect on terrestrial planet formation.

\section{Giant Planet Migration}

The extrasolar planets detected thus far (again, see Mayor \& Queloz 1995; Marcy \& Butler 1996; Butler et al. 1999; Marcy et al. 2001) have apparently moved from their birth locations in the outer nebula and now reside in rather unusual orbits, with small semi-major axes $a$ and large eccenticities $\epsilon$. An important challenge for planet formation theories is to account for this migration phenomenon. During planet migration, planetplanet scattering is effective at pumping up the orbital eccentricities but is inefficient at moving planets inward (e.g., Adams \& Laughlin 2003). Disk torques are effective at moving planets, but inefficient at increasing their eccentricities (for an opposing view, see Ogilvie \& Lubow 2003; Goldreich \& Sari 2003). A promising mechanism to account for both the semi-major axes $a$ and eccentricities $\epsilon$ of the observed extra-solar planets combines dynamical relaxation of two planets with inward forcing driven by tidal interactions with a circumstellar disk (Adams \& Laughlin 2003; Moorhead \& Adams 2004). The disk exerts a torque on the outer planet, which moves inward and interacts with the inner planet. These planet-planet scattering interactions tend to pump up the eccentricities of both planets. Since these systems are highly chaotic, the outcomes depend sensitively on 
the initial conditions and the results must be described in terms of distributions. Nevertheless, the resulting distributions of orbital elements ( $a$ and $\epsilon$ ) for the surviving planets are in reasonable agreement with the observed distributions (see Figure 2).

We have conducted a comprehensive numerical study of this migration scenario using two planets and an exterior disk (with 8000 simulations thus far). In these experiments, the two planets are placed on widely spaced orbits around a solar mass star. The inner planet starts with a period of 1900 days; the initial period of the outer planet is larger by an irrational factor so the planets do not start out in resonance. The planet masses are drawn independently from two different planetary mass distributions (denoted here as the IMF). The first IMF is a random distribution with the planet mass sampled from the range $0 \leqslant m_{P} \leqslant 5 m_{J}$; the second IMF is a log-random distribution sampled from the range $-1 \leqslant \log _{10}\left[m_{P} / m_{J}\right] \leqslant 1$. The outer planet is tidally influenced by the circumstellar disk and is gradually driven inwards. The simulations include three additional effects: (1) The angular momentum exchange between the disk and planet damps the orbital eccentricity (e.g., Agnor \& Ward 2002) of the outer planet. Since current estimates of this damping time scale give divergent results, we adopt a parametric approach and study the effect of different eccentricity damping time scales drawn from the range 0.1 Myr $\leqslant \tau_{\text {ed }} \leqslant 1$ Myr. (2) The force equations include relativistic corrections, which drive the periastron of both planetary orbits to precess and tend to move the planets away from resonance. (3) The simulations include energy loss due to tidal interactions between the planets and the star (e.g., Papaloizou \& Terquem 2001).

The simulations are integrated until only one planet remains or the integration time reaches a fiducial scale of $1 \mathrm{Myr}$. The evolutionary trend is as follows: The planets start out of resonance, but the outer planet is forced inward by the dissipative term until the planets enter into a mean motion resonance (usually the 3:1 resonance because of the starting conditions). The two planets then migrate inwards together, near resonance, but planetary interactions tend to increase the eccentricity of both orbits. The growing eccentricities drive the planets to exhibit ever-larger departures from the resonant condition. Sometimes the outer planet passes through the 3:1 resonance and then becomes held up with a period ratio of $2: 1$. The eccentricities increase in chaotic fashion until the system (usually) becomes unstable, i.e., until one of the planets is ejected or accreted by a star (or the planets collide). After a planet is lost, however, the orbit of the surviving planet continues to evolve as long as the disk is present. To account for this additional evolution, we assume that the disk has a randomly chosen lifetime (with $\tau_{\text {disk }} \leqslant 1 \mathrm{Myr}$ ) and correct the orbital elements of the surviving planet for energy dissipation and eccentricity damping over this time scale.

The orbital elements of the surviving planets display a distribution of values and this distribution is in reasonable agreement with observations. Figure 2 shows the resulting orbital elements in the $a-\epsilon$ plane for two ensembles of theoretical simulations and for the observed extrasolar planets (with the data taken from the California and Carnegie Planet Search website www.exoplanets.org). The open triangles depict the results for planet masses chosen according to the linear IMF, the open squares show the results for the log-random IMF, and the stars represent the orbital elements of the observed planets. To leading order, all three distributions fill the entire portion of the $a-\epsilon$ plane shown here. Upon closer inspection, however, one sees that the theoretical simulations tend to overproduce eccentricity relative to the observed distribution. Over much longer time scales (the $\sim 3$ Gyr lifetimes of the primary stars, rather than the $\sim 1$ Myr migration times integrated here), tidal interactions between the planets and their parental stars can lead to circularization of the orbits. The solid lines in Figure 2 delimit the portion of the $a-\epsilon$ plane for which this effect is important (where the two lines represent the 


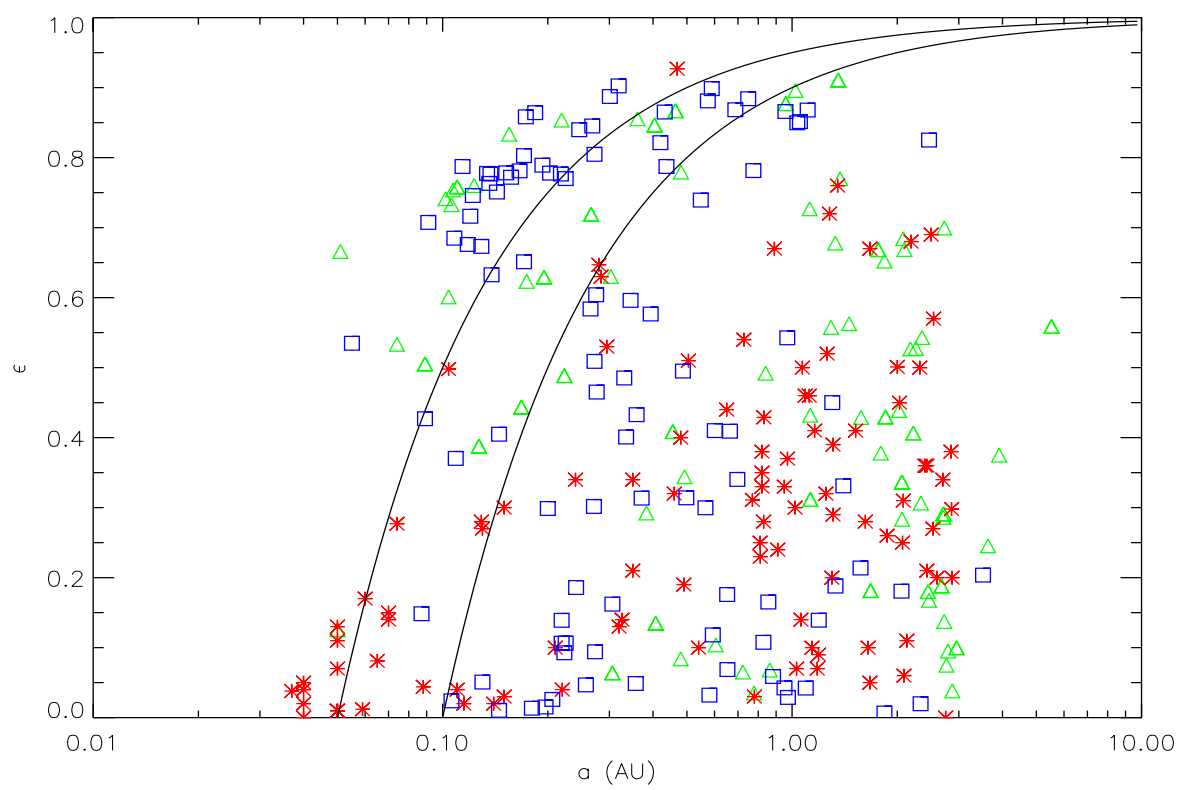

Figure 2. The $a-\epsilon$ plane for the observed population of extra-solar planets and the surviving planets in solar systems starting with two planets surrounded by a circumstellar disk (from Moorhead \& Adams 2004). The star symbols represent the observed planetary orbits. The open triangles show the surviving planets for theoretical simulations using a linear IMF. The open squares show the surviving planets for simulations using a log-random IMF. As shown here, this planet migration process results in a distribution of orbital elements. The theoretical model (with multiple planets and a disk) produces distributions of orbital parameters in reasonable agreement with the observed distribution.

variation due to the possible range in the planet's $Q$-value: see, e.g., Wu \& Goldreich 2002). With this correction for tidal circularization, the theoretical distributions are in decent agreement with the observed distribution of orbital elements.

The observed distribution of planetary mass is close to a log-random distribution. Although the planets of lowest mass tend to be those that are ejected, we find that the final mass distribution for simulations started with a log-random IMF are in good agreement with the observed mass distribution. For this log-random IMF, the numerical experiments end with about $33 \%$ ejections, $33 \%$ accretion events, and $3 \%$ collisions. The remaining simulations reach the stopping time of $1 \mathrm{Myr}$, corresponding to depletion of the circumstellar disk, without losing a planet. As a result, about one third of the systems are predicted to have multiple planets, a fraction that is somewhat higher than that of the observational sample (although additional planets might be found in the future). As expected, the outer planets are more likely to be ejected, whereas the accreted planets are almost exclusively the inner planets. As mentioned above, the ejected planets tend to be the lighter ones: The mean mass of the ejected planets is about $\left\langle m_{P}\right\rangle \approx 1.2 m_{J}$, nearly four times smaller than the mean mass of the ejectees (with $\left\langle m_{P}\right\rangle \approx 4.4 m_{J}$ ). The average time for the first planet to be ejected is about $0.5 \mathrm{Myr}$, comparable to the viscous damping time of $0.3 \mathrm{Myr}$. Finally, we note that the mean ejection speed is about $5 \mathrm{~km} / \mathrm{s}$, a result that has consequences for future observations of freely floating planets (Moorhead \& Adams 2004). 


\section{Stability of Earth-like Planets in Binary Systems}

Most stars have companions: The majority of solar type stars reside in binary systems (Abt 1983) and thus have stellar companions. In addition, recent discoveries of extrasolar planets show that Sun-like stars often have planetary mass companions (e.g., Butler et al. 1999; Marcy et. al 2001). The distributions of orbital parameters for binary star systems are relatively well known (DM91) and the orbital parameters for extrasolar planets are under intense study. If terrestrial planets form in these solar systems, the companions can affect their prospects for long term orbital stability. This section addresses the issue of planetary stability with the overall goal of estimating the fraction of solar systems that allow an Earth-like planet to remain stable over the current age of our Sun (we note that a great deal of previous work has been done, e.g., Pilat-Lohinger \& Dvorak 2002).

To study the stability of Earth-like planets in binary systems, we have performed large numbers ( 100,000 to date) of three-body simulations (David et al. 2003; Fatuzzo et al. 2004). Specifically, we consider the possible ejection of an Earth-like planet that starts in a circular orbit with radius 1 AU around its parent star, with a stellar or planetary companion that acts as a source of gravitational perturbations. Through long term dynamical interactions with the companion, the orbital elements of the Earth-like planet evolve, generally in chaotic fashion (Wisdom \& Holman 1991), until the planet is ejected from the system. For the sake of definiteness, we use a solar mass primary and a planet with one Earth mass. The mass $M_{C}$, eccentricity $\epsilon$, and semi-major axis $a$ of the companion body are then specified for each run. We consider numerical experiments with the same binary orbital parameters $\left(M_{C}, a, \epsilon\right)$ and a random distribution for the remaining (angular) orbital elements. In our initial set of simulations (David et al. 2003), the inclination angle was set to $i=0$ so the systems are co-planar; ongoing work is exploring the effects of $i \neq 0$ (Fatuzzo et al. 2004; see also Pilat-Lohinger et al. 2003). For each set of initial conditions, we integrate the system forward in time until Earth is ejected, or it collides with either star.

For simulations with given binary properties $\left(M_{C}, a, \epsilon\right)$, the ejection time $\tau_{\mathrm{ej}}$ varies with the choices of the remaining orbital elements. The systems are highly chaotic and this variation is not smooth. Figure 3 shows that the ejection time displays a log-normal distribution for an ensemble of different realizations of the same underlying problem, i.e., the same $\left(M_{C}, a, \epsilon\right)$ and a random sampling of the remaining orbital parameters. These systems thus display a distribution of ejection times with two important properties: The width of the distribution is substantial and the distribution is $\log$-normal (so that $\log \tau_{\text {ej }}$ is the relevant variable for doing statistics). The distribution of ejection times shown in Figure 3 corresponds to one point in the parameter space $\left(M_{C}, a, \epsilon\right)$. Since each point in the space corresponds to a distribution of ejection times, one must perform multiple realizations of the three-body problem for each point in question. Suppose, for example, that each point $\left(M_{C}, a, \epsilon\right)$ has the same width $\sigma \approx 0.5$ for its distribution of $\log \tau_{\text {ej. }}$. To determine the ejection time itself to 1 percent accuracy, one needs about 2625 different realizations of the problem.

As mentioned earlier, nearly two-thirds of solar-type stars live in multiple systems (Abt 1983). To estimate the fraction of binaries that allow for stable Earth-like orbits, we must combine our exploration of planetary stability with the observed distributions of binary parameters (DM91). Our initial survey of parameter space (David et al. 2003) indicates that the ejection time of an Earth-like planet is a steeply increasing function of the binary periastron $p$ (see also Chambers et al. 1996, Holman \& Wiegart 1999). To a good working approximation, the ejection time varies with periastron according to the exponential law $\tau_{\text {ej }}=\tau_{0} \exp \left[(p-1) / \ell_{0}\right]$, where the length scale $\ell_{0}$ and time scale 
$\tau_{0}$ depend on the companion mass $M_{C}$ and where are lengths are given in AU (David et al. 2003). Although the numerical simulations are only carried out to $10-100 \mathrm{Myr}$, the steepness of this time scale function allows us to extrapolate the ejection times to the age of the solar system. To obtain a conservative extrapolation, we use the lower end of the range of ejection times for each value of periastron $p$ and use the results for the largest companion mass $M_{C}$. This procedure indicates that an Earth-like planet can remain stable in a binary system if the periastron $p>7 \mathrm{AU}$, so we need to estimate the fraction of binaries with $p>7 \mathrm{AU}$. By integrating over the relevant fraction of binary parameter space $\left(M_{C}, a, \epsilon\right)$ using the results from DM91 and equation [2.1], we estimate that 50 percent of binary systems allow Earth-like planets to remain stable for the current age of the solar system (taken to be $4.6 \mathrm{Gyr}$ ).

\section{Conclusions}

This paper presents a collection of recent results concerning planet formation (Quintana et al. 2004), planet migration (Moorhead \& Adams 2004; Adams \& Laughlin 2003), and the longer term stability of planetary systems (David et al. 2003). Our findings can be summarized as follows:

During terrestrial planet formation in binary systems, we have shown that effectively equivalent starting conditions lead to a distribution of final solar system properties. In spite of the wide distribution of outcomes, however, we can extract general trends. For example, we find that the extent of terrestrial planet systems, as measured by the semimajor axis of the outermost planet, depends primarily on binary periastron $p_{b}$ (Figure 1). Combining our simulation results with the observed distribution of binary parameters, we find that about 40 percent of binary systems are wide enough (specifically, with periastron $p_{b} \geqslant 19 \mathrm{AU}$ ) so that terrestrial planet formation is relatively unaffected.

Next we have explored a promising mechanism for giant planet migration which includes a circumstellar disk and multiple planets. The disk exerts torques on the outermost planet, drives it inward, and thereby decreases its semi-major axis $a$; the inner planet often becomes locked in resonance with the outer planet and is also driven inward. During the migration epoch, scattering interactions between the planets are effective at increasing the eccentricities of both orbits. In these systems, the resulting distributions of orbital elements (e.g., in the $a-\epsilon$ plane) for the surviving planets are in reasonable agreement with the observed distributions (Figure 2).

Finally, we have considered the longer term stability of planetary systems. Binary companions can disrupt planetary orbits, and the ejection time for Earth-like planets in habitable orbits displays a wide distribution with nearly a log-normal form (Figure 3). Nonetheless, the mean ejection time varies (almost) exponentially with the binary periastron and sufficiently wide systems allow planets to remain stable over long spans of time. Again combining our numerical results with the observed distributions of binary parameters, we find that nearly 50 percent of binary systems allow Earth-like planets to survive over the current (4.6 Gyr) age of the solar system.

The results from this set of numerical investigations emphasize a general aspect of solar system dynamics. For a given set of initial conditions, none of the subproblems studied here actually has an answer. Instead, every set of effectively equivalent starting conditions leads to a full distribution of possible outcomes. The process of terrestrial planet formation leads to a distribution of planet number, planet masses, and planetary orbits. Similarly, the result of planet migration is a distribution of orbital elements for the surviving planets. Even the seemingly simple question - how long can an Earth-like planet survive in a particular binary system - has a distribution of answers (due to the 


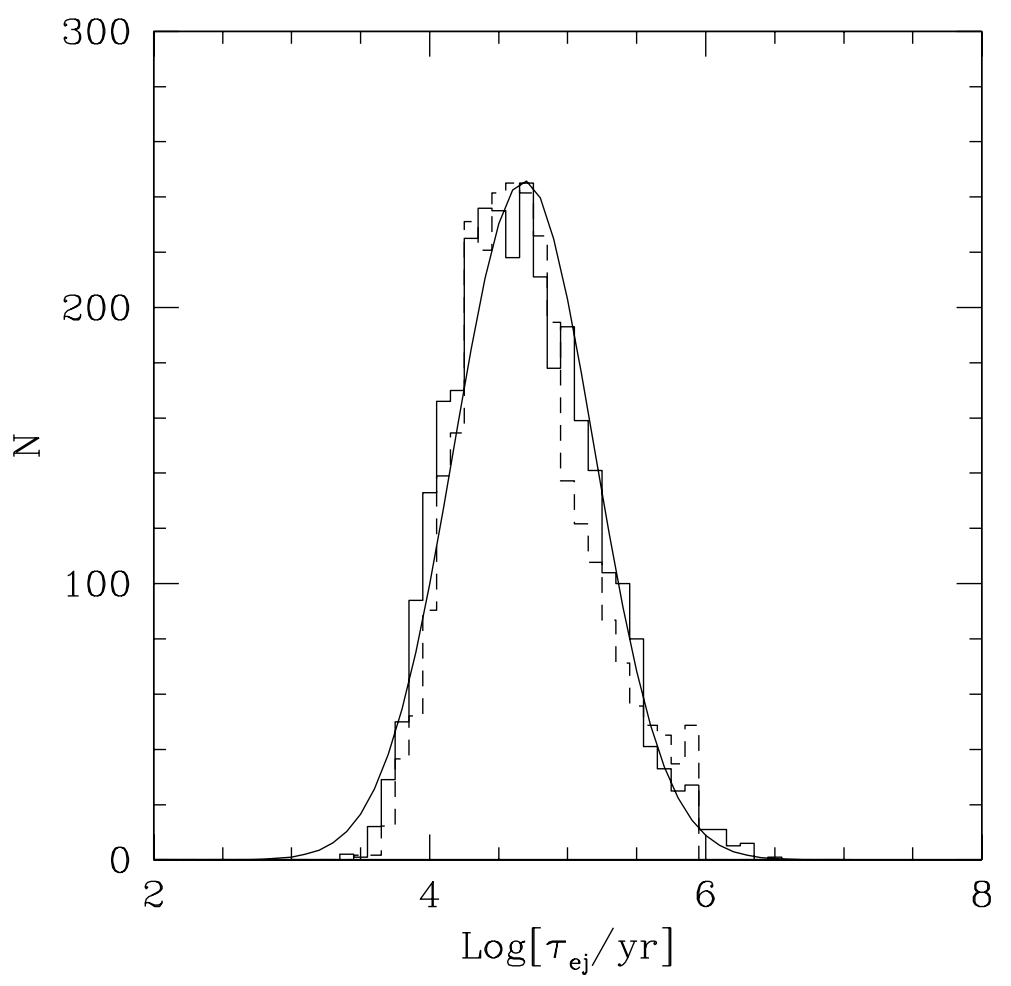

Figure 3. The distribution of ejection times for an Earth-like planet in a binary, using different realizations of the same system (from David et al. 2003). In this set of the experiments, the binary companion has mass $M_{C}=0.1 M_{\odot}$, eccentricity $\epsilon=0.5$, semi-major axis $a=5 \mathrm{AU}$, and inclination angle $i=0$. The solid histogram shows the distribution of ejection times resulting from the B-S code (for a random sampling of the remaining orbital elements). The dashed histogram shows the corresponding distribution of ejection times resulting from the symplectic code (again, for a random sampling of orbital elements). The smooth curve shows a log-normal distribution with the same peak value and width as the computed distributions. Notice that the distributions predicted by the two numerical codes are similar and that both have a log-normal form (with the same width and peak location). Because the width of this distribution is substantial, any determination of ejection times must consider a large number of realizations of the underlying problem in order to adequately sample the distribution.

dependence of the ejection time on the starting angular phases of the bodies). As a result, the dynamical systems involved in planet formation, migration, and longer term evolution display sensitive dependence on their initial conditions, one of the signposts of chaotic behavior. In the face of such chaos, the outcome of the planet formation/migration process must generally be described in terms of distributions of possible results. In spite of this complication, however, astronomical results can be obtained: In the context of planet migration, we have shown that our theoretical mechanism provides roughly the same distribution of orbital elements as the observational sample. In addition, we have shown here that 40 percent of binary systems are wide enough to not disrupt the formation of 
terrestrial planets and that about 50 percent of binary systems are wide enough to allow Earth-like planets to remain stable for the age of the solar system.

\section{Acknowledgements}

This contribution describes work done with a number of colleagues and I would like to especially thank the following collaborators: Eva David, Marco Fatuzzo, Greg Laughlin, Jack Lissauer, Althea Moorhead, and Elisa Quintana. In addition I would like to thank the conference organizers and local hosts, especially Zoran Knežević and Milan Ćirković, for their hospitality. This work was supported at the University of Michigan by the Michigan Center for Theoretical Physics and by NASA through the Terrestrial Planet Finder Mission (NNG04G190G) and the Astrophysics Theory Program (NNG04GK56G0).

\section{References}

Abt, H. 1983, ARAEA 21, 343

Adams, F. C., \& Laughlin, G. 2003, Icarus 163, 290

Agnor, C. B., \& Ward, W. R. 2002, Astrophys. J. 567, 579

Butler, R. P., et al. 1999, Astrophys. J. 526, 916

Chambers, J. E. 1999, Mon. Not. R. Astron. Soc. 304, 793

Chambers, J. E. 2001, Icarus 152, 205

Chambers, J. E., Quintana, E. V., Duncan, M. J., \& Lissauer, J. J. 2002, Astron. J. 123, 2884

Chambers, J. E., Wetherill, G. W., \& Boss, A. P. 1996, Icarus 119, 261

David, E.-M., Quintana, E. V., Fatuzzo, M., \& Adams, F. C. 2003, Publ. Astron. Soc. Pacific 115,825

Duquennoy, A., \& Mayor, M. 1991, Astron. Astrophys. 248, 485 (DM91)

Fatuzzo, M., Adams, F. C., Doering, C. R., \& Gaubin, R. 2004, in preparation

Goldreich, P., \& Sari, R. 2003, Astrophys. J. 585, 1024

Holman, M. J., \& Wiegart, P. A. 1999, Astron. J. 117, 621

Levison, H. F. \& Agnor, C. 2003, Astron. J. 125, 2692

Levison, H. F., Lissauer, J. J., \& Duncan, M. J. 1998, Astron. J. 116, 1998

Lissauer, J. J. 1993, ARAESA 31, 129

Lissauer, J. J., Quintana, E. V., Chambers, J. E., Duncan, M. J., \& Adams, F. C. 2004, in: Gravitational Collapse: From Massive Stars to Planets, in press

Marcy, G. W., \& Butler, R. P. 1996, Astrophys. J. 464, L147

Marcy, G. W., et al. 2001, Astrophys. J. 556, 296

Mayor, M., \& Queloz, D. 1995, Nature 378, 355

Moorhead, A. V., \& Adams, F. C. 2004, Icarus, submitted

Ogilvie, G. I., \& Lubow, S. H. 2003, Astrophys. J. 587, 398

Papaloizou, J.C.B., \& Terquem, C. 2001, Mon. Not. R. Astron. Soc. 325, 221

Pilat-Lohinger, E., \& Dvorak, R. 2002, Cel. Mech. Dyn. Astron. 82, 143

Pilat-Lohinger, E., Funk, B., \& Dvorak, R. 2003, Astron. Astrophys., 400, 1085

Quintana, E. V. 2004, PhD Thesis, Physics Department, University of Michigan

Quintana, E. V., Adams, F. C., \& Lissauer, J. J. 2004, in preparation

Wisdom, J., \& Holman, M. 1991, Astron. J. 102, 1528

Wolszczan, A., \& Frail, D. A. 1992, Nature 355, 145

Wu, Y., \& Goldreich, P. 2002, Astrophys. J. 564, 1024 